\title{
The design, analysis and application of mouse clinical trials in oncology drug development
}

\author{
Sheng Guo ${ }^{1 *} \mathbb{D}$, Xiaoqian Jiang ${ }^{1}$, Binchen Mao ${ }^{1}$ and Qi-Xiang Li $i^{2,3^{*}}$
}

\begin{abstract}
Background: Mouse clinical trials (MCTs) are becoming wildly used in pre-clinical oncology drug development, but a statistical framework is yet to be developed. In this study, we establish such as framework and provide general guidelines on the design, analysis and application of MCTs.

Methods: We systematically analyzed tumor growth data from a large collection of PDX, CDX and syngeneic mouse tumor models to evaluate multiple efficacy end points, and to introduce statistical methods for modeling MCTs.

Results: We established empirical quantitative relationships between mouse number and measurement accuracy for categorical and continuous efficacy endpoints, and showed that more mice are needed to achieve given accuracy for syngeneic models than for PDXs and CDXs. There is considerable disagreement between methods on calling drug responses as objective response. We then introduced linear mixed models (LMMs) to describe MCTs as clustered longitudinal studies, which explicitly model growth and drug response heterogeneities across mouse models and among mice within a mouse model. Case studies were used to demonstrate the advantages of LMMs in discovering biomarkers and exploring drug's mechanisms of action. We introduced additive frailty models to perform survival analysis on MCTs, which more accurately estimate hazard ratios by modeling the clustered mouse population. We performed computational simulations for LMMs and frailty models to generate statistical power curves, and showed that power is close for designs with similar total number of mice. Finally, we showed that MCTs can explain discrepant results in clinical trials.
\end{abstract}

Conclusions: Methods proposed in this study can make the design and analysis of MCTs more rational, flexible and powerful, make MCTs a better tool in oncology research and drug development.

Keywords: PDX, CDX, Syngeneic model, Mouse clinical trials, Linear mixed models, Survival analysis, Statistical power, Biomarker

\section{Background}

Cancer is a heterogeneous disease with intra- and intertumor genomic diversity that determines cancer initiation, progression and treatment. The understandings of cancer biology and the development of therapeutics have been aided greatly by a variety of mouse tumor models,

\footnotetext{
*Correspondence: guosheng@crownbio.com; henryli@crownbio.com ${ }^{1}$ Crown Bioscience Inc., Suzhou Industrial Park, 218 Xinghu Street, Jiangsu 215028, China

${ }^{2}$ Crown Bioscience, Inc, 3375 Scott Blvd, Suite 108, Santa Clara, CA 95054, USA

Full list of author information is available at the end of the article
}

including cell line-derived xenografts (CDXs), patient derived-xenografts (PDXs), genetically engineered mouse models (GEMMs), cell line- or primary tumor-derived homografts in syngeneic mice and so on (reviewed by [1-4]). These models differ in their generation, host and tumor genomics and biology, availability, and research utilizations. For example, immunotherapies are tested in immunocompetent models such as GEMMs and syngeneic models.

Past decades witnessed the accelerated creation, distribution, profiling and characterization of mouse tumor

(C) The Author(s). 2019 Open Access This article is distributed under the terms of the Creative Commons Attribution 4.0 International License (http://creativecommons.org/licenses/by/4.0/), which permits unrestricted use, distribution, and 
models [5-10]. The abundant collections made it possible to conduct the so-called "mouse clinical trials (MCTs)", in which a panel of mouse models, dozens to hundreds, are used to evaluate therapeutic efficacy, discover/validate biomarkers, study tumor biology and so on. MCTs demonstrated faithful clinical predictions in multiple studies [6,11-15]. While most reported MCTs used PDXs, MCTs using other mouse models, such as syngeneic models, are now widely performed as well.

Because of their resemblance to clinical trials, MCTs are often analyzed by methods for clinical trials. For example, overall survival (OS) and progression-free survival (PFS) are estimated by tumor volume increase, Cox proportional hazards models are used for survival analysis, response categories are defined by tumor volume change and objective response rate (ORR) is calculated $[6,13,16]$. However, MCTs differ from clinical trials in many ways. (1) In an oncology clinical trial, a patient is enrolled in only one arm, while in a MCT, multiple mice bearing tumor from the same mouse model are made so that mice can be placed in all arms. Mice from the same mouse model capture intra-tumor heterogeneity for tumor growth and drug response, and mice from different mouse models capture inter-tumor heterogeneity. Measurement error can be quantified when multiple mice are used in each arm. Furthermore, since there are mice of same mouse models in both arms, they themselves can serve as control across arms for better measurement of drug efficacy. (2) tumor volumes are routinely measured every few days; (3) mouse models are usually characterized with genomic/ pharmacology/histopathology annotations; (4) MCTs are done in labs that reduces/removes various noise and inconvenience encountered in clinical trials, such as dropouts, long trial time and concomitant medication.

In this study, we combine empirical data analysis, statistical modeling and computational simulations to address some key issues for MCTs, including the determination of animal numbers (number of mouse models and number of mice per mouse model), statistical power calculation, quantification of efficacy difference between mice/mouse models/drugs, survival analysis, biomarker discovery/validation with and beyond simple efficacy readouts, handling of mouse dropouts, missing data and difference in tumor growth rates, study of mechanisms of action (MoA) for drugs. We will also show MCTs can explain discrepant clinical trial results.

\section{Methods}

Mouse models, studies and transcriptomic profiling

The establishment of mouse models and the conduct of mouse efficacy studies were described previously [17-19]. Briefly, for PDX models, freshly resected patient tumors were sliced into roughly $3 \times 3 \times 3 \mathrm{~mm}^{3}$ chunks and engrafted subcutaneously on the flanks of immunocompromised mice (BALC/c, NOD/SCID, NOG, etc.). Tumor growth was monitored by a caliper twice a week to establish the first passage of a PDX model. Tumor was harvested for next round of engraftment when it reached $500-700 \mathrm{~mm}^{3}\left(1 / 2\right.$ length $\times$ width $\left.^{2}\right)$. A series of engraftment produced subsequent passages of the model. For CDX and syngeneic models, cell suspension $\left(0.1-5 \times 10^{6}\right.$ cell/mouse) was injected into immunocompromised mice and immunocompetent mice (C57BC/6, BALB/c, etc.), respectively, to induce tumor. Pharmacological dosing started when a tumor was normally $100-300 \mathrm{~mm}^{3}$, tumor volume was measured twice a week until the tumor was reaching $3000 \mathrm{~mm}^{3}$, by then the mouse was euthanized. All animal studies were conducted at Crown Bioscience SPF facility under sterile conditions and were in strict accordance with the Guide for the Care and Use of Laboratory Animals of the National Institutes of Health. Protocols of all studies were approved by the Committee on the Ethics of Animal Experiments of Crown Bioscience, Inc. (Crown Bioscience IACUC Committee). Mouse models and cell lines were profiled by RNA-seq on Illumina HiSeq series platforms by certified service providers, as previously described [7].

\section{Categorical efficacy endpoints in mouse studies}

Four categorical endpoint methods were evaluated, including the Response Evaluation Criteria In Solid Tumors (RECIST) criteria [20], a 3-category or 3-cat method [13], the 4-response mRECIST criterion [6], and a 5-category or 5-cat method [16]. Briefly, the RECISTbased criterion categorizes drug responses into complete response (CR), partial response (PR), stable disease (SD) and progressive disease (PD) based on relative tumor volume, or RTV, at a later day relative to treatment initiation day (CR: $\mathrm{RTV}=0$, PR: $0<\mathrm{RTV} \leq 0.657$, SD: $0.657<\mathrm{RTV} \leq 1.728$, PD: RTV > 1.728). Metastasis is not considered because it rarely occurs in subcutaneous implantation. The 3-cat method classifies response into $\mathrm{PD}, \mathrm{SD}$ and objective response (OR) based RTV as well (OR: $\mathrm{RTV} \leq 0.65$, PD: $\mathrm{RTV} \geq 1.35$, SD: $0.65<$ RTV < 1.35). The mRECIST method considers tumor growth kinetics 10 days after treatment initiation and classifies responses into $\mathrm{CR}, \mathrm{PR}, \mathrm{SD}$ and $\mathrm{PD}$ using two RTV-based quantities: best response and best average response. The 5-cat method classifies responses into maintained CR (MCR), CR, PR, SD and PD based on RTV (PD: RTV $>0.50$ during the study period and RTV $>1.25$ at end of study, SD: RTV > 0.50 during the study period and $\mathrm{RTV} \leq 1.25$ at end of study, PR: $0<\mathrm{RTV} \leq 0.50$ for at least one time point, CR: $\mathrm{RTV}=0$ for at least one time point, MCR: 
$\mathrm{RTV}=0$ at end of study). In the definitions of MCR and $\mathrm{CR}$, we also use RTV $=0$ to designate disappearance of measurable tumor mass to replace the convention $\left(\mathrm{TV}<0.10 \mathrm{~cm}^{3}\right)$ used in Houghton et al., 2007. For all 4 methods, the admissive initial tumor volume is $50 \sim 300 \mathrm{~mm}^{3}$. Objective response is defined as $\mathrm{OR}, \mathrm{CR}+\mathrm{PR}, \mathrm{MCR}+\mathrm{CR}+\mathrm{PR}$ in the 3-cat, RECIST/mRECIST and 5-cat methods, respectively.

\section{Continuous efficacy endpoints in mouse studies}

We briefly describe 4 continuous endpoints here. (a) Progression-free survival (PFS) is defined as tumor volume doubling time and obtained by linear intrapolation on tumor growth data. Specifically, if the PFS is between day $d_{1}$ and day $d_{2}$, then it is $d_{1}+\left(d_{2}-d_{1}\right)\left(2 T V_{0}-T V_{1}\right) /$ $\left(T V_{2}-T V_{1}\right)$ where $T V_{1}, T V_{2}$ and $T V_{0}$ are tumor volumes at $d_{1}, d_{2}$ and treatment initiation day. (b) RTV ratio is the ratio of RTV between drug group and vehicle group at a specific day $d$ and equals $\mathrm{RTV}_{\mathrm{t}} / \mathrm{RTV}_{\mathrm{c}}$, where $\mathrm{RTV}_{\mathrm{t}}$ is the relative tumor volume between day $d$ and treatment initiation day for the drug treatment group, and $\mathrm{RTV}_{\mathrm{c}}$ is accordingly defined for the vehicle group. (c) Tumor growth inhibition (TGI) has several definitions, it can be defined as $1-\mathrm{RTV}_{\mathrm{t}} / \mathrm{RTV}_{\mathrm{c}}$, or as $1-\Delta \mathrm{T} / \Delta \mathrm{C}$ where $\Delta \mathrm{T}$ and $\Delta \mathrm{C}$ are tumor volume changes relative to initial volume for drug group and vehicle group, respectively, at a specific day. (d) The ratio of growth rates between drug group and vehicle group is defined as $k_{\mathrm{t}} / k_{\mathrm{c}}$ where $k_{\mathrm{t}}$ and $k_{\mathrm{c}}$ are the growth rates obtained by modeling tumor growth data for the two groups by Eq. 1. More general, we can introduce a new endpoint called AUC ratio, which reduces to ratio of growth rates when tumor grows under exponential kinetics (Fig. S5). Unique treatment models with at least 10 mice were used to calculate continuous endpoints, including 621 unique treated PDXs, 739 CDXs and 438 syngeneic models.

\section{Modeling tumor growth}

Tumor growth under exponential kinetics is modeled by

$$
T V_{d}=T V_{0} e^{k d}
$$

Where $T V_{O}$ is the initial tumor volume, $T V_{\mathrm{d}}$ is the tumor volume at day $d$, and $k$ is the tumor growth rate. A logarithmic transformation gives

$$
\ln T V_{d}=\ln \left(T V_{0}\right)+k d
$$

\section{Linear mixed models for the cisplatin dataset}

A general model can be specified for tumor volume, in $\log$ scale, at day $t$ for mouse $i$ within $\operatorname{PDX} j$ as follows:

$$
\begin{aligned}
\log T V_{t i j}= & \beta_{0}+\beta_{1} \times \text { Day }_{t}+\beta_{2} \times \text { Day }_{t} \\
& \times \text { CancerTypeGA }_{j}+\beta_{3} \times \text { Day }_{t} \\
& \times \text { CancerTypeLU }_{j}+\beta_{4} \times \text { Day }_{t} \\
& \times \text { Treatment }_{i j}+\beta_{5} \times \text { Day }_{t} \\
& \times \text { CancerTypeGA }_{j} \times \text { Treatment }_{i j} \\
& +\beta_{6} \times \text { Day }_{t} \times \text { CancerTypeLU }_{j} \\
& \times \text { Treatment }_{i j}+u_{0 j}+u_{1 j} \times \text { Day }_{t} \\
& +u_{(0 i \mid j)}+u_{(1 i \mid j)} \times \text { Day }_{t}+\varepsilon_{t i j}
\end{aligned}
$$

LU is lung cancer, GA is gastric cancer and ES is esophageal cancer. The model uses vehicle in ES as the reference. There are 6 fixed effects: $\beta_{0}$ for the intercept, $\beta_{1}$ for the time slope, $\beta_{2}$ and $\beta_{3}$ quantify the growth rate difference of GA and LU with respect to ES, $\beta_{4}$ measures cisplatin effect, $\beta_{5}$ and $\beta_{6}$ measures if GA and LU respond differently to cisplatin. The model also has 5 random effects, including the residual $\varepsilon_{t i j}$. In a MCT, we view the cohort of PDXs as random samples from a PDX or patient population, therefore, they have different growth rates, which is modeled by random effect $u_{1 j}$ associated with the time slope. Similarly, we model growth difference for mice within a PDX by the random effect $u_{1 i \mid j}$. Mice and PDX may have different starting tumor volumes, modeled by the two random effects on intercept $u_{0 j}$ and $u_{0 i \mid j}$.

\section{Power calculation based on computational simulation}

Power calculation was based on parameters (e.g., variance and covariance of random effects) estimated from fitting the cisplatin dataset by a LMM:

$$
\begin{aligned}
\log T V_{t i j}= & \beta_{0}+\beta_{1} \times \text { Day }_{t}+\beta_{2} \times \text { Day }_{t} \\
& \times \text { Treatment }_{i j}+u_{0 j}+u_{1 j} \times \text { Day }_{t} \\
& +u_{(0 i \mid j)}+u_{(1 i \mid j)} \times \text { Day }_{t}+\varepsilon_{t i j}
\end{aligned}
$$

At significance level $\alpha=0.05$, we obtained power curves by simulations for $\beta_{2} / \beta_{1}=-0.1$ to -0.9 , that is, drug treatment reduces tumor growth rate by 10 to $90 \%$.

\section{Additive frailty models for survival analysis}

In the additive frailty model, the hazard function for the $j$-th mouse of the $i$-th mouse model is given by

$$
h_{i j}(t)=h_{0}(t) \exp \left(u_{i}+\left(w+v_{i}\right) T_{i j}+\beta^{\mathrm{T}} X_{i}\right)(5)
$$

where $h_{0}(t)$ is the baseline hazard function. Parameter $u_{i}$ is the random effect (the first frailty term) associated with the $i$-th mouse model that captures its characteristic growth, thus survival behavior, without drug treatment. Parameter $v_{i}$ is the random effect (the second frailty term) associated with the $i$-th mouse model that depicts its drug response. Parameter $w$ measures the drug treatment effect on all mouse models. $T_{i j}$ is the treatment variable and equals 0 for the vehicle treatment and 1 for the drug treatment; $X_{i}$ is a vector for the mouse model's covariates, e.g., cancer type and genomic 
features; $\beta^{\mathrm{T}}$ is the parameter vector quantifying the fixed effects of the covariates. The two random effects $u_{i}$ and $v_{i}$ assume a bivariate normal distribution with zero means, variance $\sigma^{2}$ and $\tau^{2}$, and covariance $\rho \sigma \tau$. If the two random effects $u_{i}$ and $v_{i}$ are removed, the model reduces to the Cox proportional hazards model. Model fitting was done by the $\mathrm{R}$ package frailtypack (version 2.12.6), assuming Weibull distribution for the hazard function [21].

\section{Linear mixed models for the biomarker discovery}

The following LMM is used for single-gene biomarker discovery by fitting efficacy data from a MCT:

$$
\begin{aligned}
\log T V_{t i j}= & \beta_{0}+\beta_{1} \times \text { Day }_{t}+\beta_{2} \times \text { Day }_{t} \\
& \times \text { Gene }_{j}+\beta_{3} \times \text { Day }_{t} \times \text { Treatment }_{i j} \\
& +\beta_{4} \times \text { Day }_{t} \times \text { Gene }_{j} \times \text { Treatment }_{i j} \\
& +u_{0 j}+u_{1 j} \times \text { Day }_{t}+\mathbf{u}_{(0 i \mid j)}+u_{(1 i \mid j)} \\
& \times \text { Day }_{t}+\varepsilon_{t i j}
\end{aligned}
$$

In this model, Gene is a covariate for the genomic status (expression, mutation, copy number variation, etc.) of a gene.

\section{Gene list enrichment analysis}

A list of top ranked genes were used as input to the Enrichr web server (http://amp.pharm.mssm.edu/Enrichr/) for their enrichment in the "Reactome 2016" pathway database and in the "GO Biological Process 2018" database [22]. Adjusted $p$-values were used to rank enriched pathways and biological processes.

\section{Protein-protein interaction network analysis}

A list of top ranked genes were analyzed for proteinprotein interactions in the STRING database (version 10.5 at https://string-db.org) [23]. Default settings were used except the value for "minimum required interaction score" changed from "medium confidence (0.400)" to "high confidence (0.700)".

\section{Results}

\section{Determining number of mice for categorical responses}

We collected tumor volume data under drug treatment for 26127 mice from 2883 unique treatment PDXs, 11139 mice from 1219 unique treatment CDXs, and 5945 mice from 637 unique treatment syngeneic models. A unique treatment model is a mouse model treated by a drug in a study. Every unique treatment has at least 8 mice. Categorical drug response was determined by 4 methods (see Materials and Methods), and we illustrate the results using the mRECIST criteria, which classifies drug response into 4 categories: complete response (CR), partial response (PR), stable disease (SD), and progressive disease (PD). For each unique treatment model, its response is the majority response of all mice. We observed that individual mouse responses matched the majority response most often for PD: $90 \%$ for PDXs, 95\% for CDXs and syngeneic models (Fig. 1a-c). The other 3 response categories exhibit lower concordance, particularly so for syngeneic models. Of the 10 unique treatment syngeneic models classified as CR, only half of the mice had complete response as well, while $17 \%$ of mice were PD and resistant to treatment. Such polarized response pattern is observed in the other 3 methods, too (Additional file 1: Figure S1-S3). Large variance exists for all 4 response categories. For example, only about $70 \%$ of individual responses matched the majority response for a third of the 107 unique treatment PDX models categorized as CR, although the average is $83 \%$.

Measurement accuracy increases with number of mice. We randomly sampled $\mathrm{n}(n=1,3,5,7)$ mice from all the mice in a treatment and obtained a majority response, which was then compared with the actual majority response. The procedure was repeated for 1,000 times to generate statistical results (Fig. 1d-f). Accuracy increases with mouse number for all 4 categories, and their unweighted average is highest in CDXs, which is slightly higher than PDXs, while syngeneic models have much lower accuracy (Fig. 1g). Therefore, more mice are needed for syngeneic models to achieve similar accuracy as PDXs/CDXs. For example, accuracy is comparable between syngeneic studies with 5 mice per model and PDX/CDX studies with 1 mouse per model. Similar patterns are also seen in the other 3 methods (Additional file 1: Figure S1-S3).

All the 4 methods categorize responses based on relative tumor volume (RTV) at a later day to treatment initiation day, but differ in specific thresholds. As such, a unique treatment model can be categorized differently. We found that there is a good overlapping for unique treatment models classified as objective response between the 4 methods (Fig. 1h-j), and their objective response rates (ORR) are similar. (Additional file 1: Table S1). Nevertheless, there are many models only unique to some methods as OR, cautioning methodspecific bias and applicability. For example, the mRECIST considers averaging tumor reduction for a period of time, therefore, a unique treatment model can be classified as PD even though tumor completely disappears at end of study (Additional file 1: Figure S4).

\section{Determining number of mice for continuous responses}

Drug efficacy can be measured by continuous responses, some are direct adaption of clinical endpoints (e.g., PFS and OS), others are unique to mouse studies that use data from both vehicle and drug treatment groups (e.g., RTV ratio between drug and vehicle groups). We calculated the estimation errors of PFS and RTV ratio 

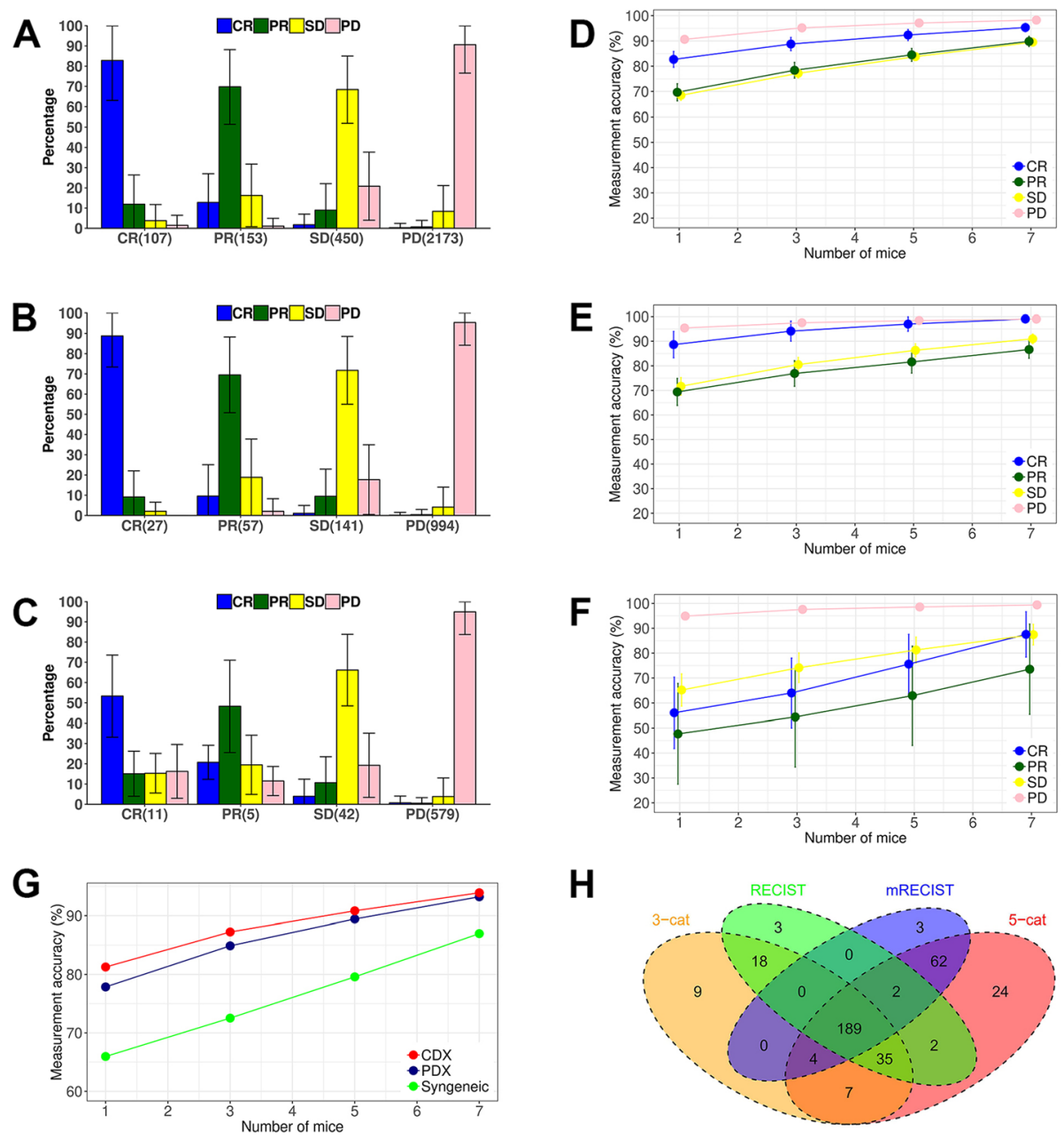

H
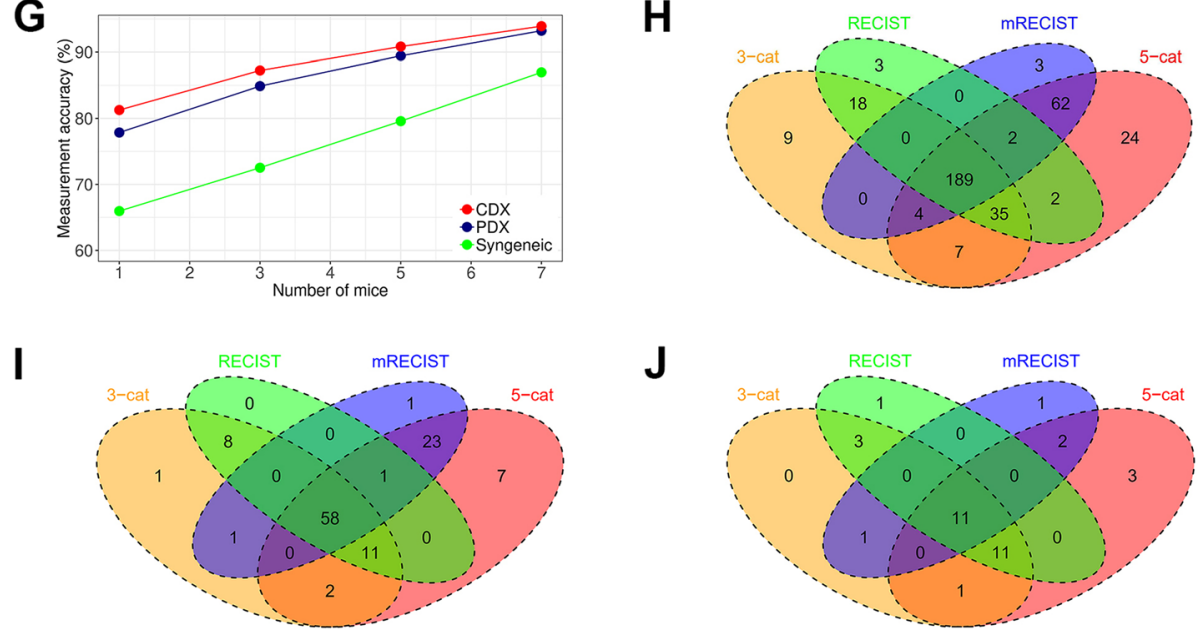

Fig. 1 Mouse number and measurement accuracy of categorical responses defined by the mRECIST criteria. (a-c): individual mouse response and majority response in PDX $(\mathbf{a}), \operatorname{CDX}(\mathbf{b})$ and syngeneic models $(\mathbf{c}), x$ axis is the number of majority response from 4 response categories (CR: complete response, PR: partial response, SD: stable disease, PD: progressive disease.), y axis is the percentage of individual mouse response relative to the majority (average \pm s.d.). There are 26,127 mice in 2,883 unique treatment PDX models, 11,139 mice in 1,219 unique treatment CDX models, and 5,945 mice in 637 unique treatment syngeneic models. Each unique treatment model had at least 8 mice. (d-g): measurement accuracy increases with number of mice for PDX (d), CDX (e) and syngeneic models (f). For each unique treatment model, the majority response of $\mathrm{n}(n=1,3,5,7$ in $\mathrm{x}$ axis) randomly sampled mice was obtained to see if it agreed with the actual majority response. The procedure was repeated 1,000 times to obtain the accuracy_-percentage of times (average \pm s.d.) that they agreed—for the 4 response categories, whose unweighted average is shown in $(\mathbf{g})$. (h-j): Venn diagram showing the overlap of unique treatment PDX models classified as objective response by 4 categorical methods in PDX (h), CDX (i), and syngeneic models (j). Objective response is OR in the 3-cat method, CR + PR in the mRECIST and RECIST methods, MCR + CR + PR in the 5-cat method

computed from $\mathrm{n}(n=1$ to 9$)$ mice randomly sampled from the $\geq 10$ mice in a study, and obtained the quantitative relationship between estimation errors and mouse numbers (Fig. 2). For each n, we obtained the empirical cumulative density function (ECDF) with respect to percentage error of PFS estimate for PDX, CDX and syngeneic models (Fig. 2a-c), and with respect to the absolute error of RTV ratio estimate for the three types 

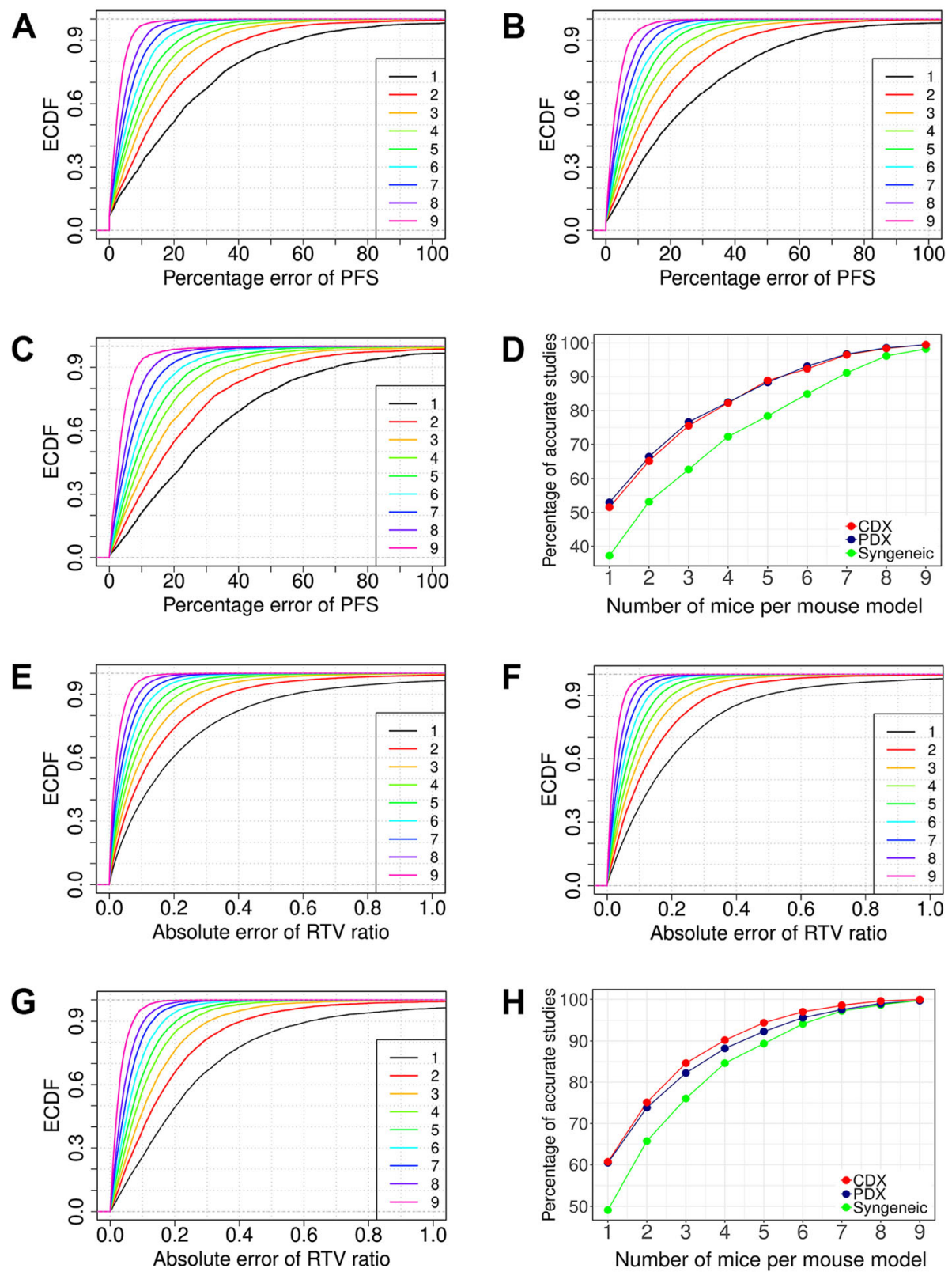

Fig. 2 Determining mouse numbers for continuous responses. (a-c): Progression-free survival, or PFS, calculated from $n$ mice $(n=1$ to 9) randomly sampled from a unique treatment model with at least 10 mice shows relative deviation to the PFS calculated from all mice in PDX (a), CDX (b), and syngeneic models (c), $x$ axis is the percent error of PFS, and $y$ axis is the empirical cumulative density function (ECDF) estimated from the random samplings for each n. Percent error of PFS decreases with increased number of mice, and the error is larger for syngeneic models than PDXs/CDXs. (d): Percentages of unique treatment models with percent error less than $20 \%$ in the 3 types of mouse models. (e-g): RTV ratio between drug and vehicle groups, calculated from $n$ mice ( $n=1$ to 9) randomly sample from a study with at least 10 mice in both drug and vehicle groups, shows deviation to the RTV ratio calculated from all mice in both groups in PDX (e), CDX (f), and syngeneic models (g), $x$ axis is the absolute error, and $y$ axis is the empirical cumulative density function (ECDF) estimated from the random samplings for each $n$. Absolute error of RTV ratio decreases with increased number of mice, and the error is larger for syngeneic models than PDXs/CDXs. (h): Percentages of studies with absolute error less than 0.2 in the 3 types of mouse models

of models (Fig. 2e-g). Large estimation errors are inherent to small sample sizes, particularly so for syngeneic models. For example, percent error of PFS is greater than $20 \%$ for $63 \%$ syngeneic mice and for about half of
PDX/CDX mice (Fig. 2d). Estimation errors are reduced sharply by addition of more mice when $\mathrm{n}$ is small. For RTV ratio, 3 mice in both drug and vehicle group already lift mice with absolute error $<0.2$ from $60 \%$ to 
above $80 \%$ for PDXs/CDXs (Fig. 2h). Similar results hold for other continuous endpoints as well (Additional file 1: Figure S5).

\section{Modeling MCTs as clustered longitudinal studies}

It is convenient to measure drug efficacy by a categorical or continuous endpoint, but those approaches also suffer from loss of information and other drawbacks. For example, it is somewhat arbitrary to choose a day to calculate RTV ratio and TGI; it adds logistic burden to match mice with comparable tumor volume at treatment initiation day [24]; it is difficult to deal with mouse dropouts. These shortcomings can be overcome by modeling MCTs as clustered longitudinal studies, in which a cluster is consisted of all mice of a mouse model so they share genomic profile and have more similar drug response. Each mouse is in a longitudinal study. It can be shown that tumor growth in majority of mice follows exponential kinetics (Additional file 1: Figure S6). Therefore, we can model the clustered longitudinal studies by a 3-level linear mixed model (LMM) on the logtransformed tumor volumes (logTV) and day (Fig. 3a). There are covariates associated with mouse models such as cancer type and genomic features, which can be used for examining efficacy difference on cancers and for discovering predictive biomarkers.

We use one example to demonstrate the modeling of MCTs by LMMs for efficacy evaluation and comparison. In this MCT, cisplatin-a chemotherapy drug-was administrated to 42 PDXs $(4 \mathrm{mg} / \mathrm{kg}$, weekly dosing for 3 weeks), including 13 esophageal cancers (ES), 21 gastric cancers (GA) and 8 lung cancers (LU), each PDX with 5 to 9 mice (Additional file 1: Figure S7). We fit the efficacy data by a LMM (Eq. 3 in Materials and Methods), which explicitly models tumor growth rate heterogeneity and drug response heterogeneity at both PDX level and mouse level. Model fitting is satisfactorily (Table 1, Additional file 1: Figure S8). We conclude that (1) under vehicle treatment, tumor in GA grows slightly faster than ES, while tumor growth is much faster in LU; (2) cisplatin has comparable efficacy on the 3 cancers $(p$ values for $\beta_{5}$ and $\beta_{6}$ are $>0.05$ ). The results can be readily visualized from the mean growth curves for the 3 cancers under (Fig. 3b).

\section{Statistical power and sample size determination in MCTs}

Much like clinical trials, rational design of MCTs requires statistical power calculation and sample size determination-number of mouse models and number of mice per mouse model. We demonstrate this under the LMM framework with the following assumptions (1) a balanced $n: n$ design in which there are $n(\geq 1)$ mice in both drug and vehicle groups, and (2) a 21-day trial with tumor volume measured at treatment initiation and then twice every week to produce 8 data points for every mouse. Drug efficacy is measured by how much drug treatment slows down tumor growth $\left(\beta_{2} / \beta_{1}\right.$ in Eq. 4). Power curves were obtained by computational simulations based on parameters obtained from fitting the cisplatin dataset by Eq. 4 (Fig. 3c).

We observed that if the number of PDXs is the same, more mice per PDX confer better statistical power. For example, to achieve $80 \%$ power, we need about 28 PDXs for the 1:1 design (1 mouse each in the vehicle and drug treatment groups), and 11 PDXs for the 3:3 design (3 mice each in the vehicle and drug treatment groups). More importantly, statistical power is comparable for designs with similar number of total mice. For example, when the drug efficacy is $20 \%$, that is, the drug reduces tumor growth rate by $20 \%$, the following designs all achieve $90 \%$ power at 0.05 significance level: 36 PDX with 1:1 design, 19 PDXs with 2:2 design, 13 PDXs with 3:3 design, 10 PDXs with 4:4 design, and so on. However, it is important to note that such designs with similar statistical power and total number of mice have different biological implications. A design with a larger number of PDX but fewer mice or even one mouse per PDX can give better representation and measurement of inter-tumor heterogeneity, while a design with a smaller number of PDX but more mice per PDX sacrifices such inter-tumor heterogeneity to give more accurate measurement of drug efficacy for each PDX. It depends on study aims to choose a design. For example, we likely prefer a design with more PDX each with fewer mice for biomarker discovery because it would give us a broader representation of inter-tumor heterogeneity and more genomic datasets to work with. In the extreme case, we can use the 1:1 design if there are many PDXs at disposal-the $1 \times 1 \times 1$ approach [6], in which Gao et al. showed that the 1:1 design is effective in biomarker assessment and efficacy evaluation. But for biomarker validation, we may use a design with a limited number of selected PDX models that are predicted to be responsive or resistant, and each PDX should have a relatively high number of mice so that the efficacy measurement is accurate enough to gauge the effectiveness of the biomarker. The design also are constrained by available resource, for example, when there is only a limited number of suitable PDXs, e.g., PDXs carrying a particular mutation or PDXs of a specific subtype, we can increase the number of mice per PDX to boost statistical power.

We also observed that fewer PDXs are needed for a more potent drug to reach same statistical power. For example, to achieve $80 \%$ statistical power at 0.05 significance level by the $3: 3$ design, we need about 40,11 , and 5 PDXs for drugs with 10, 20, and 30\% efficacy, respectively. When a drug is potent enough, all n:n designs achieve high power with very small number of PDXs. In 


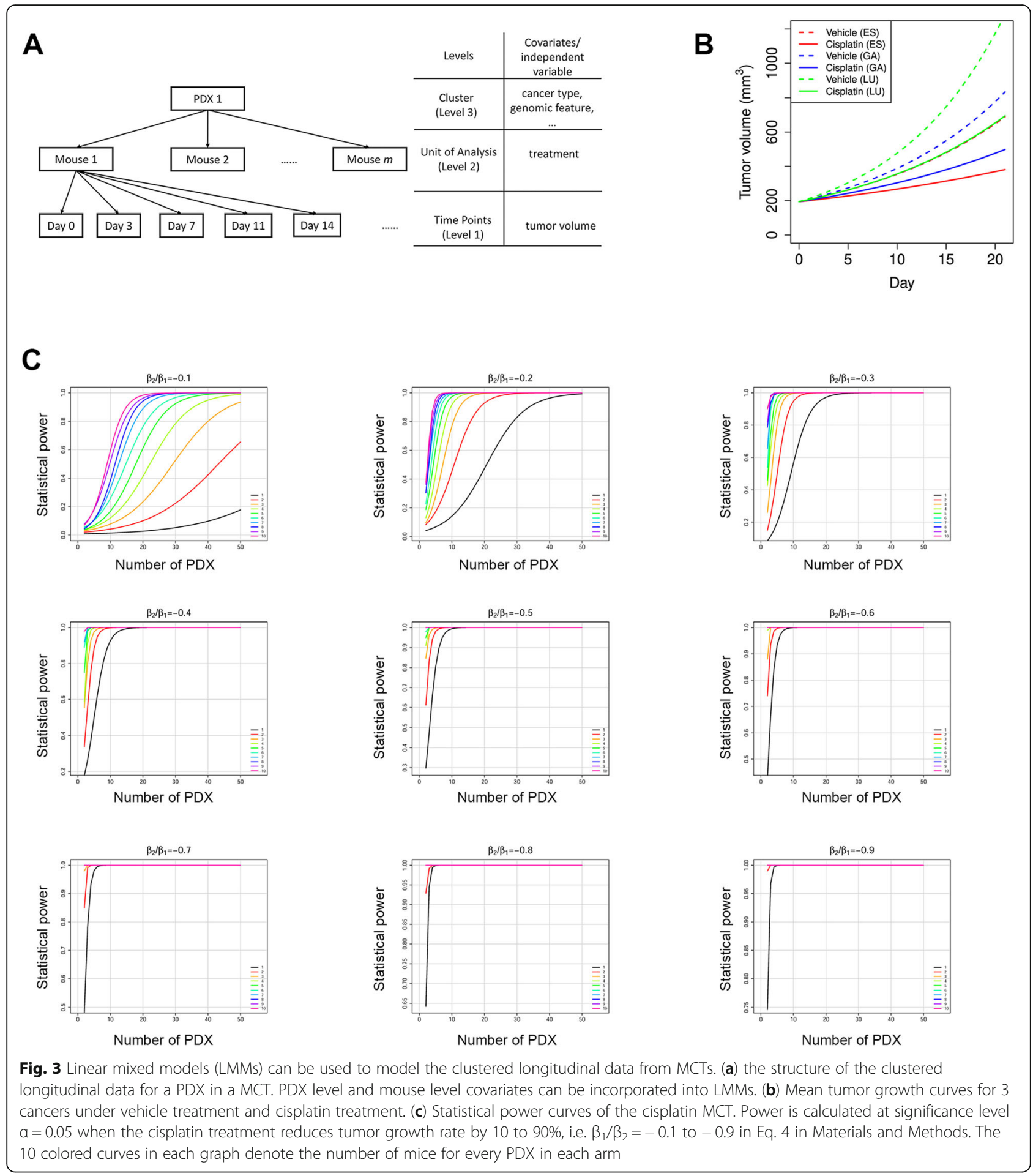

such cases, we use a good number of PDXs not for statistical power but for better representation of tumor heterogeneity.

\section{Survival analysis in MCTs}

In clinical trials, patient survival is usually assumed to be independent of each other. In MCTs, this assumption no longer holds because mice are now clustered within PDXs, and mice of same PDX tend to have more similar survival time, while their survival time between treatments is highly correlated (Fig. 4a). Further, PDXs can vary greatly in growth rate (or hazard) and drug response (Additional file 1: Figure S9). Therefore, we use an additive frailty model to model the heterogeneity on 
Table 1 Parameters estimated for the LMM (Eq. 3) of the cisplatin dataset

\begin{tabular}{lll}
\hline Fixed-Effect Parameters & Estimate $^{*}$ & p-value \\
\hline$\beta_{0}$ (Intercept) & $5.2641(0.0257)$ & 0 \\
$\beta_{1}$ (Day) & $0.0605(0.0043)$ & $1.5 \mathrm{E}-43$ \\
$\beta_{2}$ (Day $\times$ CancerTypeGA) & $0.0091(0.0055)$ & 0.098 \\
$\beta_{3}$ (Day $\times$ CancerTypeLU) & $0.0297(0.0071)$ & $2.8 \mathrm{E}-5$ \\
$\beta_{4}$ (Day $\times$ Treatment) & $-0.0282(0.0031)$ & $1.2 \mathrm{E}-19$ \\
$\beta_{5}$ (Day $\times$ CancerTypeGA $\times$ Treatment) & $0.0037(0.0039)$ & 0.35 \\
$\beta_{6}$ (Day $\times$ CancerTypeLU $\times$ Treatment) & $-0.0011(0.0052)$ & 0.84 \\
\hline
\end{tabular}

*parameters estimated by the REML method in the $\mathrm{R}$ nIme package

hazard and drug efficacy under the clustered population structure of MCTs (see Eq. 5 in Materials and Methods). The additive frailty model is an extension of the Cox proportional hazards model wildly used in clinical trials. It has two frailty terms, the first one $u_{i}$ quantifies PDX growth rate heterogeneity and the second one $v_{i}$ measures drug response heterogeneity.

We use the cisplatin MCT to illustrate the utilization of the additive frailty model. Overall survival (OS) is defined as tumor volume tripling time. We fit the cisplatin MCT dataset by Eq. 5, and observed that both frailty terms are significant larger than 0 (Wald test $p$-value $<0.05$ ), proving that the PDXs grow at different rate and had different responses to cisplatin. In fact, the first frailty term $u_{i}$ is negatively correlated with tumor growth rate in the vehicle group, as expected $\left(R^{2}=0.85\right.$, Fig. $\left.4 b\right)$.

Drug efficacy can be estimated more accurately by excluding the influence of tumor growth heterogeneity and considering drug response heterogeneity, which is measured by the second frailty term $v_{i}$. Indeed, the hazard ratio (HR) is estimated to be 0.21 (95\% CI: $0.15-0.31$ ), much smaller than that obtained from the Cox proportional hazards model, which gives $\mathrm{HR}=0.36$ (95\% CI, 0.28-0.46) (Fig. 4c). These results show that without considering PDX heterogeneity, drug effect can be severely misestimated.

We performed statistical power analysis for the survival analysis by assuming the n:n designs and using parameters estimated from the cisplatin MCT with Weibull hazard functions (Fig. 4d). Like in LMMs, statistical power is similar for designs with similar total number of mice.

\section{Biomarker discovery in MCTs}

Genomic correlation to cetuximab efficacy in solid tumors has been well documented [13], and we previously reported a MCT for a cohort of 20 gastric cancer PDXs, each with 3-10 mice in the vehicle and cetuximab treatment arms. We found that EGFR expression to be a predictive biomarker for cetuximab on gastric cancer [19]. The cohort is now expanded to 27 PDXs (Additional file 1: Figure S10). We observed a strong correlation between EGFR expression and drug efficacy measured by tumor growth inhibition or TGI (Fig. 5a). When all 18586 genes were ranked from high to low by the absolute value of correlation coefficient between their expression and TGI, EGFR is ranked 157 out of all these genes, demonstrating that such simple methods in biomarker discovery can yield many false positives with seemingly better predictivity than the true biomarker.

We used a LMM that explicitly models a gene's effect on tumor growth to fit the efficacy data (Eq. 6 in Materials and Methods). EGFR stands out as the most significant gene and its $p$-value, being $1.5 \times 10^{-23}$, is at least five orders of magnitude smaller than all other genes (Fig. 5b). EGFR as a predictive biomarker for cetuximab on gastric cancer is supported by a phase 2 clinical trial [25] and a phase 3 clinical trial with data re-interpretation (Additional file 1: Figure S11) [26]. This study shows that simple analysis can produce many false positive hits to hamper biomarker discovery, especially when a drug target is unknown or there are off-target effects, while the more sophisticated LMM method can be superior in biomarker discovery.

\section{Mechanism of action study in MCTs}

MCTs are used for drug efficacy evaluation and biomarker discovery, the latter can be facilitated by a better understanding of a drug's mechanism of action (MoA), which helps identify relevant genes, pathways and gene sets, and remove false positive genes that could have higher statistical significance, i.e. lower $p$-values, in some analysis. Biomarkers constructed from genes selected this way have explicit biological relevance and oftentimes are preferred.

With the readily available genomic and efficacy data from a MCT, MoA studies can be readily performed. Like in biomarker discovery, simple categorical and continuous endpoints, as a gross summery of efficacy, have various drawbacks. For example, the 4 categorical methods only measure efficacy in drug treatment group, ignoring the relative drug-to-vehicle efficacy. RTV ratio and TGI are dependent on calculation day and tumor growth rate (Additional file 1: Figure S12). Again, we can use LMM for a better study of MoA, as shown by the example below.

Irinotecan is a DNA topoisomerase I inhibitor that interrupts cell cycle in the S-phase by irreversibly arresting the replication fork, therefore causing cell death [27]. We conducted a MCT for 16 PDXs (Additional file 1: Figure S13), each PDX with 3 to 10 mice. We modeled the effect of gene expression on drug efficacy by a LMM 


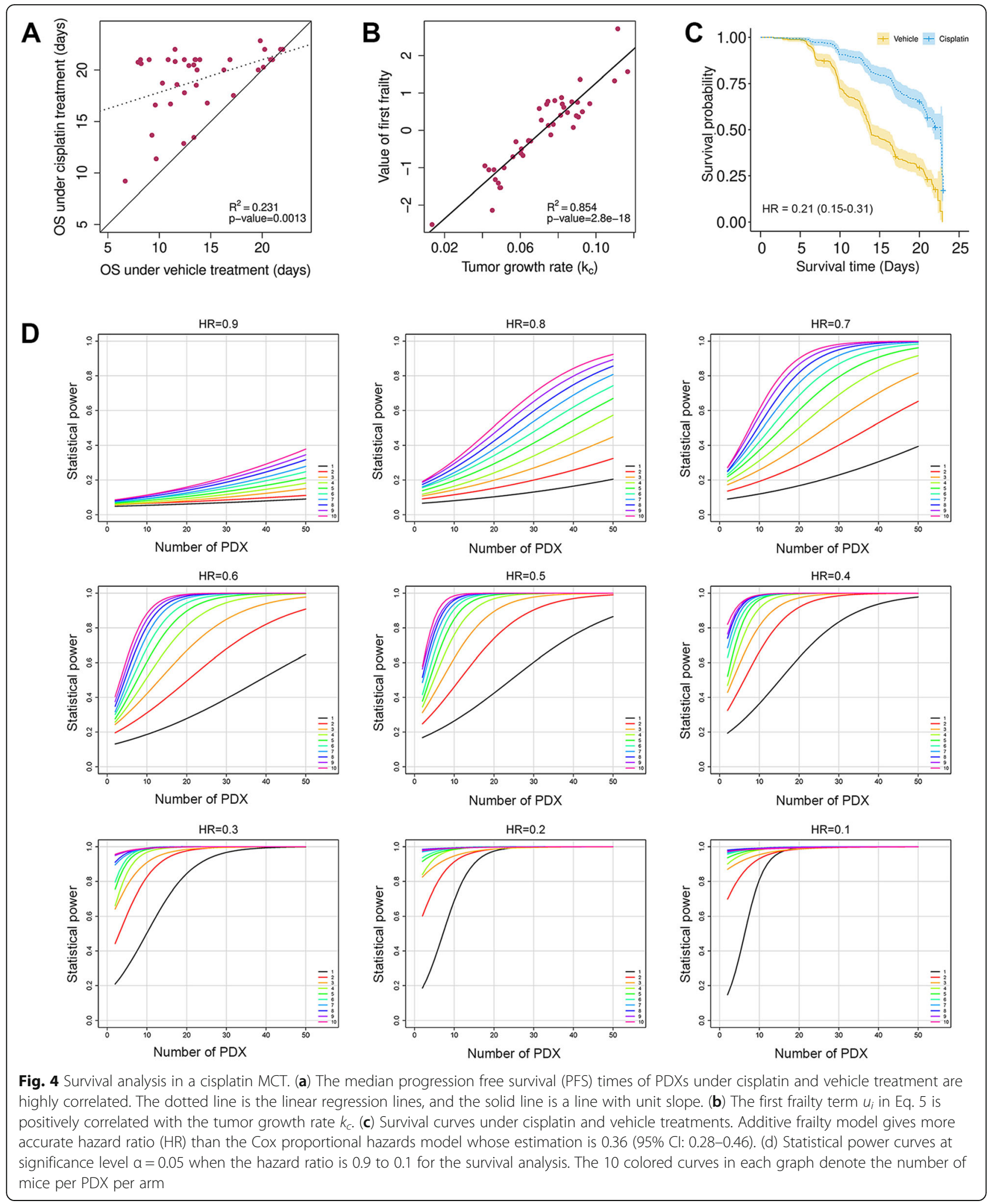

(Eq. 6). Top ranked genes were highly enriched for the cell cycle pathway R-HSA-160170 in the Reactome 2016 database (Fig. 5c), and for DNA replication initiation
(Gene Ontology annotation GO: 0006270) (Fig. 5d), which perfectly reveals the MoA for irinotecan. A highly connected protein-protein interaction network for cell 
A
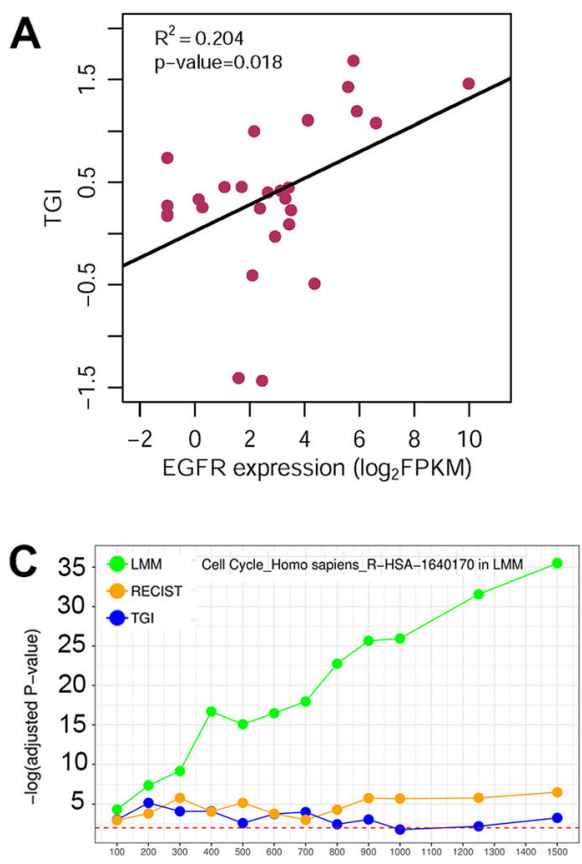

Number of top genes

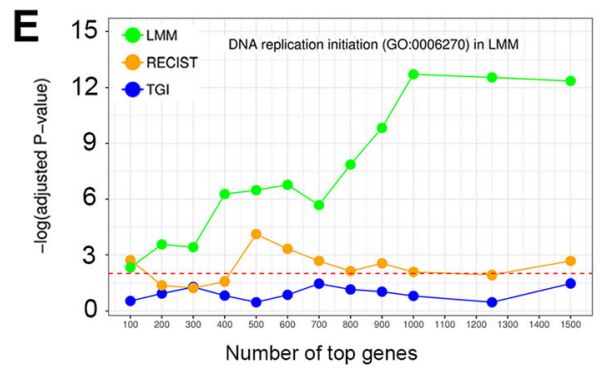

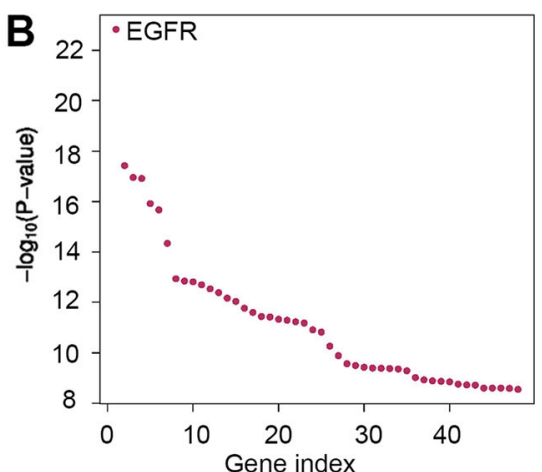

D

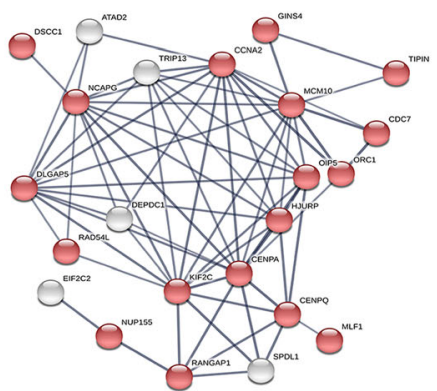

$\mathbf{F}$

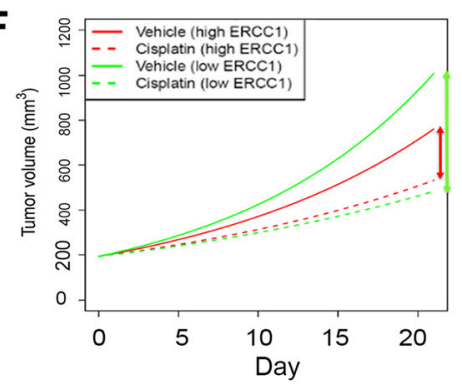

Fig. 5 Biomarker discovery and MoA study in MCTs. (a-b): A MCT of 27 gastric cancer PDXs treated with cetuximab. EGFR is ranked 157th among all genes based on Spearman rank correlation between EGFR expression and TGI (a), but is the top gene in predicting cetuximab efficacy based on a linear mixed model (LMM) (b). (c-e): A MCT of 16 PDXs treated with intraperitoneal injection of Irinotecan (100 mg/kg, once per week for 2 to 3 weeks). (c): R-HAS-160170, the cell cycle pathway in Reactome2016 database, is consistently ranked as the most enriched pathway with 100 to 2000 top genes selected by a LMM, superior to top genes selected by methods based on categorical endpoints (e.g., RECIST, Table S2) and continuous endpoints (e.g., TGI). (d): DNA replication initiation (GO: 0006270) is the most enriched GO term based on top genes selected by the LMM. (e): A highly enriched protein-protein interaction network ( $p$-value $<10^{-16}$ ) consisted of 23 genes in the top 100 genes selected by the LMM. Red-colored nodes are ones involved in cell cycle (GO: 0007049). Dashed horizontal lines in (c-d) denotes p-value $=0.01$. (f): Mean tumor growth curves for PDXs with highest and lowest ERCC1 mRNA expression in a MCT of 21 gastric PDXs treated by cisplatin

cycle is also identified from the 100 top ranked genes (Fig. 5e). In contrast, endpoint based methods are far less insightful (Fig. 5c-d, Additional file 1: Table S2-S4).

\section{MCTs can explain paradoxical clinical trial results}

Conflicting clinical trial reports exist regarding the role of ERCC1 expression in predicting cisplatin treatment on gastric cancer: some claimed that patients benefit more from low ERCC1 expression [28-32], some stated the opposite [33-35], while still others found no connection at all [36].
In a previous section, we described a cisplatin MCT which included 21 gastric cancer PDXs. We fit the tumor volume data by Eq. 6. Parameter $\beta_{2}$ quantifies how ERCC1 expression affects tumor growth when there is no drug intervention, as seen from the vehicle growth curves (Fig. 5f). Parameter $\beta_{4}$ evaluates how ERCC1 expression impacts cisplatin's efficacy on tumor growth, as seen by comparing the cisplatin growth curves with corresponding vehicle growth curves. These two parameters are at comparable magnitude but with opposite signs $\left(\beta_{2}=-0.0155\right.$ and $\left.\beta_{4}=0.0136\right)$. Therefore, when ERCC1 expression gets higher, tumor grows 
slower, but the benefit of cisplatin treatment is smaller as well (Fig. 5f).

In a clinical trial, patients with low/negative ERCC1 expression would have worse prognosis if they were not treated, and they could benefit more from cisplatin treatment. With treatment, their prognosis is improved, but whether it is better than the prognosis of ERCC1 high/positive patients is undetermined and depends on the trial population, hence we saw conflicting study conclusions.

\section{Discussion}

MCTs are population-based efficacy trials mimicking human trials. Multiple mice are usually used per mouse model per arm to improve accuracy of efficacy measurement. For example, Bertotti et al. used 6 mice per PDX per arm in a two-arm MCT with 85 colorectal cancer PDXs to identify HER2 as a therapeutic target in Cetuximab-resistant colorectal cancers [11]. It may also be feasible to use one mouse per model per arm when there is a large number of mouse models, which compensate the loss of measurement accuracy on individual mice $[6,8,37,38]$. Caution must be exercised to use this approach though, when the number of mouse models is small, or high measurement accuracy of individual mouse models is mandated, or response varies greatly among mice of same mouse models, as commonly observed for immunotherapeutic agents on syngeneic models. Syngeneic models, unlike PDX or CDX that are immunodeficient mouse tumor models, have intact immune system, which likely is the source for large variation of drug response among mice within a syngeneic model, because individual mice can vary greatly in tumor immunity including the levels of T-cell infiltration, Th1 cytokine expression, and immunogenicity [39].

Our study established theoretic foundations for the design and analysis of MCTs. We first investigated tumor growth kinetics. Many complex mathematical models were used to describe tumor growth [40], but might not be particularly advantageous at the expense of more parameters and the need of more data points for model fitting. The exponential growth model is simple, interpretable and linear after a logarithmic transformation, and was shown to be adequate in most cases. Consequently, LMMs can describe nearly all MCTs, using quadratic terms of time if necessary.

We introduced additive frailty models to perform survival analysis for MCTs. The definition of PFS/OS can vary. For example, OS can be defined same as in human trials for leukemia PDXs [8]. For both LMMs and frailty models, we performed power simulations that give concrete recommendations on trial design. In particular, we answered the frequently asked questions, from a statistical perspective, on how many mouse models and how many mice per model to use, with flexible combination of the two numbers. We emphasize that it is equally important to consider the purpose of MCTs, e.g., biomarker discovery versus biomarker validation, in the study design, and designs with more PDX but fewer mice per PDX (e.g. 1:1 design) have better representation of inter-tumor heterogeneity than ones with fewer PDX but more mice per PDX (e.g., 3:3 design), but the latter gives more accurate measurement of drug efficacy. MCTs can be asymmetric, i.e., unequal numbers of mice in arms. LMMs and frailty models are flexible for covariates, for example, a fixed effect for site can be incorporated if a MCT is conducted at multiple sites.

\section{Conclusions}

In conclusion, methods proposed in this study make the design and analysis of MCTs more rational, flexible and powerful when mouse tumor models are used in oncology research and drug development.

\section{Additional file}

Additional file 1: Figure S1. Mouse number and measurement accuracy of categorical responses defined by the RECIST criteria. Figure S2. Mouse number and measurement accuracy of categorical responses defined by the 3-cat criterion. Figure $\mathbf{S 3}$. Mouse number and measurement accuracy of categorical responses defined by the 5-cat criterion. Figure S4. A unique treatment model classified as PD by mRECIST method, though tumor completely disappeared at end of study. Figure S5. AUC ratio as a continuous metric for MCTs. Figure S6. (a) Distribution of coefficient of determination between log-transformed tumor volume and day for PDX mice under vehicle treatment. Figure S7. Growth curves of 42 PDXs under vehicle treatment (a) and cisplatin treatment (b). Figure S8. Fitting diagnostics of the linear mixed model in Eq. 3 for the cisplatin MCT dataset (cf. Fig. S7). Figure S9. Tumor volume doubling time in PDXs for 10 cancers. Figure S10. Growth curves of 27 PDXs under (a) vehicle treatment and (b) cetuximab treatment (1 mg/mouse, intraperitoneal injection, once per week). Figure S11. In the EXPAND phase III trial (1), for patients with IHC score greater than $\sim 200$, the 7 patients receiving cetuximab in addition to had significantly longer (a) PFS and (b) OS than the 19 patients receiving only chemotherapies. Figure S12. TGI is a growth rate biased and time-dependent efficacy metric. Figure S13. Growth curves of 16 PDXs under (a) vehicle treatment and (b) Irinotecan treatment $(100 \mathrm{mg} / \mathrm{kg}$, intraperitoneal injection, once per week for 2-3 weeks. Table S1. Objective response rate (ORR) in 4 categorizing methods.

Table S2. Irinotecan response of 16 PDX models by 4 categorical endpoint methods. Table S3. Most enrichment pathways in Reactome 2016 database for the Irinotecan MCT. Table S4. Most enrichment terms in GO Biological Processes for the Irinotecan MCT. (PDF 2790 kb)

\footnotetext{
Abbreviations

CR: Complete response; CDX: Cell line-derived xenograft; EGFR: Epiderma growth factor receptor; ERCC: Excision repair 1 (ERCC1); ES: Esophageal cancer; GA: Gastric cancer; GEMM: Genetically engineered mouse model; HER2: Human epidermal growth factor receptor 2; HR: Hazard ratio; LMM: Linear mixed model; LU: Lung cancer; MCT: Mouse clinical trial; MoA: Mechanism of action; OR: Objective response; OS: Overall survival; PD: Progressive disease; PDX: Patient-derived xenograft; PFS: Progression-free survival; PR: Partial response; RCT: Randomized controlled trial; RECIST: Response Evaluation Criteria in Solid Tumors; RTV: Relative tumor volume; SD: Stable disease; TGI: Tumor growth inhibition
} 


\section{Acknowledgements}

The authors would like to express their gratitude to the in vivo team members at the Translational Oncology Division of Crown Bioscience, Inc. for contributing all the in vivo efficacy data.

\section{Authors' contributions}

SG and QL designed the study and wrote the manuscript, SG, XJ, BM analyzed the data. All authors have read and approved the manuscript.

\section{Funding}

Not applicable.

\section{Availability of data and materials}

Datasets used in the current study are available from the corresponding authors on reasonable request.

\section{Ethics approval and consent to participate}

All animal studies were conducted at Crown Bioscience SPF facility under sterile conditions and were in strict accordance with the Guide for the Care and Use of Laboratory Animals of the National Institutes of Health. Protocols of all studies were approved by the Committee on the Ethics of Animal Experiments of Crown Bioscience, Inc. (Crown Bioscience IACUC Committee).

\section{Consent for publication}

Not applicable.

\section{Competing interests}

This research was funded by Crown Bioscience Inc. and all authors were employees thereof at the time the study was performed. The authors declare no other competing interests.

\section{Author details}

${ }^{1}$ Crown Bioscience Inc., Suzhou Industrial Park, 218 Xinghu Street, Jiangsu 215028, China. ${ }^{2}$ Crown Bioscience, Inc, 3375 Scott Blvd, Suite 108, Santa Clara, CA 95054, USA. ${ }^{3}$ State Key Laboratory of Natural and Biomimetic Drugs, Peking University, Beijing 100191, China.

Received: 8 December 2018 Accepted: 5 July 2019

Published online: 22 July 2019

\section{References}

1. Day CP, Merlino G, Van Dyke T. Preclinical mouse cancer models: a maze of opportunities and challenges. Cell. 2015;163(1):39-53.

2. Khaled WT, Liu P. Cancer mouse models: past, present and future. Semin Cell Dev Biol. 2014;27:54-60.

3. Li QX, Feuer G, Ouyang X, An X. Experimental animal modeling for immuno-oncology. Pharmacol Ther. 2017;173:34-46.

4. Walrath JC, Hawes JJ, Van Dyke T, Reilly KM. Genetically engineered mouse models in cancer research. Adv Cancer Res. 2010;106:113-64.

5. Byrne AT, Alferez DG, Amant F, Annibali D, Arribas J, Biankin AV, Bruna A, Budinska E, Caldas C, Chang DK, et al. Interrogating open issues in cancer precision medicine with patient-derived xenografts. Nat Rev Cancer. 2017;17(4):254-68.

6. Gao H, Korn JM, Ferretti S, Monahan JE, Wang Y, Singh M, Zhang C, Schnell C, Yang G, Zhang Y, et al. High-throughput screening using patient-derived tumor xenografts to predict clinical trial drug response. Nat Med. 2015; 21(11):1318-25.

7. Guo S, Qian W, Cai J, Zhang L, Wery JP, Li QX. Molecular pathology of patient tumors, patient-derived xenografts, and Cancer cell lines. Cancer Res. 2016:76(16):4619-26.

8. Townsend EC, Murakami MA, Christodoulou A, Christie AL, Koster J, DeSouza TA, Morgan EA, Kallgren SP, Liu H, Wu SC, et al. The public repository of xenografts enables discovery and randomized phase II-like trials in mice. Cancer Cell. 2016;30(1):183.

9. Krupke DM, Begley DA, Sundberg JP, Richardson JE, Neuhauser SB, Bult CJ. The mouse tumor biology database: a comprehensive resource for mouse models of human Cancer. Cancer Res. 2017;77(21):e67-70.

10. Stewart E, Federico SM, Chen X, Shelat AA, Bradley C, Gordon B, Karlstrom A, Twarog NR, Clay MR, Bahrami A, et al. Orthotopic patient-derived xenografts of paediatric solid tumours. Nature. 2017;549(7670):96-100.

11. Bertotti A, Migliardi G, Galimi F, Sassi F, Torti D, Isella C, Cora D, Di Nicolantonio F, Buscarino M, Petti C, et al. A molecularly annotated platform of patient-derived xenografts ("xenopatients") identifies HER2 as an effective therapeutic target in cetuximab-resistant colorectal cancer. Cancer Discov. 2011;1(6):508-23.

12. Migliardi G, Sassi F, Torti D, Galimi F, Zanella ER, Buscarino M, Ribero D, Muratore A, Massucco P, Pisacane A, et al. Inhibition of MEK and PI3K/mTOR suppresses tumor growth but does not cause tumor regression in patientderived xenografts of RAS-mutant colorectal carcinomas. Clin Cancer Res. 2012;18(9):2515-25.

13. Bertotti A, Papp E, Jones S, Adleff V, Anagnostou V, Lupo B, Sausen M, Phallen J, Hruban CA, Tokheim C, et al. The genomic landscape of response to EGFR blockade in colorectal cancer. Nature. 2015;526(7572):263-7.

14. Bardelli A, Corso S, Bertotti A, Hobor S, Valtorta E, Siravegna G, SartoreBianchi A, Scala E, Cassingena A, Zecchin D, et al. Amplification of the MET receptor drives resistance to anti-EGFR therapies in colorectal cancer. Cancer Discov. 2013;3(6):658-73.

15. Yao YM, Donoho GP, Iversen PW, Zhang Y, Van Horn RD, Forest A, Novosiadly RD, Webster YW, Ebert P, Bray S, et al. Mouse PDX trial suggests synergy of concurrent inhibition of RAF and EGFR in colorectal Cancer with BRAF or KRAS mutations. Clin Cancer Res. 2017;23(18):5547-60.

16. Houghton PJ, Morton CL, Tucker C, Payne D, Favours E, Cole C, Gorlick R, Kolb EA, Zhang W, Lock $R$, et al. The pediatric preclinical testing program: description of models and early testing results. Pediatr Blood Cancer. 2007; 49(7):928-40.

17. Yang M, Shan B, Li Q, Song X, Cai J, Deng J, Zhang L, Du Z, Lu J, Chen T, et al. Overcoming erlotinib resistance with tailored treatment regimen in patient-derived xenografts from naive Asian NSCLC patients. Int J Cancer. 2013;132(2):E74-84.

18. Yang M, Xu X, Cai J, Ning J, Wery JP, Li QX. NSCLC harboring EGFR exon-20 insertions after the regulatory $C$-helix of kinase domain responds poorly to known EGFR inhibitors. Int J Cancer. 2016;139(1):171-6.

19. Zhang L, Yang J, Cai J, Song X, Deng J, Huang X, Chen D, Yang M, Wery JP, Li $S$, et al. A subset of gastric cancers with EGFR amplification and overexpression respond to cetuximab therapy. Sci Rep. 2013;3:2992.

20. Eisenhauer EA, Therasse P, Bogaerts J, Schwartz LH, Sargent D, Ford R, Dancey J, Arbuck S, Gwyther S, Mooney M, et al. New response evaluation criteria in solid tumours: revised RECIST guideline (version 1.1). Eur J Cancer. 2009;45(2):228-47

21. Rondeau V, Gonzalez JR : frailtypack: a computer program for the analysis of correlated failure time data using penalized likelihood estimation. Comput Methods Prog Biomed 2005, 80(2):154-164.

22. Chen EY, Tan CM, Kou Y, Duan Q, Wang Z, Meirelles GV, Clark NR, Ma'ayan A. Enrichr: interactive and collaborative HTML5 gene list enrichment analysis tool. BMC Bioinformatics. 2013;14:128.

23. Szklarczyk D, Franceschini A, Wyder S, Forslund K, Heller D, Huerta-Cepas J, Simonovic M, Roth A, Santos A, Tsafou KP, et al. STRING v10: protein-protein interaction networks, integrated over the tree of life. Nucleic Acids Res. 2015:43(Database issue):D447-52.

24. Laajala TD, Jumppanen M, Huhtaniemi R, Fey V, Kaur A, Knuuttila M, Aho E, Oksala R, Westermarck J, Makela S, et al. Optimized design and analysis of preclinical intervention studies in vivo. Sci Rep. 2016;6:30723.

25. Zhang $X, X u$ J, Liu H, Yang L, Liang J, Xu N, Bai Y, Wang J, Shen L. Predictive biomarkers for the efficacy of cetuximab combined with cisplatin and capecitabine in advanced gastric or esophagogastric junction adenocarcinoma: a prospective multicenter phase 2 trial. Med Oncol. 2014;31(10):226.

26. Lordick F, Kang YK, Chung HC, Salman P, Oh SC, Bodoky G, Kurteva G, Volovat C, Moiseyenko VM, Gorbunova V, et al. Capecitabine and cisplatin with or without cetuximab for patients with previously untreated advanced gastric cancer (EXPAND): a randomised, openlabel phase 3 trial. Lancet Oncol. 2013;14(6):490-9.

27. Xu Y, Villalona-Calero MA. Irinotecan: mechanisms of tumor resistance and novel strategies for modulating its activity. Ann Oncol. 2002;13(12):1841-51.

28. De Dosso S, Zanellato E, Nucifora M, Boldorini R, Sonzogni A, Biffi R, Fazio N, Bucci E, Beretta O, Crippa S, et al. ERCC1 predicts outcome in patients with gastric cancer treated with adjuvant cisplatin-based chemotherapy. Cancer Chemother Pharmacol. 2013;72(1):159-65.

29. Hirakawa M, Sato Y, Ohnuma H, Takayama T, Sagawa T, Nobuoka T, Harada K, Miyamoto H, Sato Y, Takahashi Y, et al. A phase II study of neoadjuvant combination chemotherapy with docetaxel, cisplatin, and S-1 for locally advanced resectable gastric cancer: nucleotide excision repair (NER) as potential chemoresistance marker. Cancer Chemother Pharmacol. 2013;71(3):789-97. 
30. Kwon HC, Roh MS, Oh SY, Kim SH, Kim MC, Kim JS, Kim HJ. Prognostic value of expression of ERCC1, thymidylate synthase, and glutathione S-transferase P1 for 5-fluorouracil/oxaliplatin chemotherapy in advanced gastric cancer. Ann Oncol. 2007:18(3):504-9.

31. Metzger R, Leichman CG, Danenberg KD, Danenberg PV, Lenz HJ, Hayashi K, Groshen S, Salonga D, Cohen H, Laine L, et al. ERCC1 mRNA levels complement thymidylate synthase mRNA levels in predicting response and survival for gastric cancer patients receiving combination cisplatin and fluorouracil chemotherapy. J Clin Oncol. 1998;16(1):309-16.

32. Miura JT, Xiu J, Thomas J, George B, Carron BR, Tsai S, Johnston FM, Turaga KK, Gamblin TC. Tumor profiling of gastric and esophageal carcinoma reveal different treatment options. Cancer Biol Ther. 2015;16(5):764-9.

33. Baek SK, Kim SY, Lee JJ, Kim YW, Yoon HJ, Cho KS. Increased ERCC expression correlates with improved outcome of patients treated with cisplatin as an adjuvant therapy for curatively resected gastric cancer. Cancer Res Treat. 2006;38(1):19-24.

34. Bamias A, Karina M, Papakostas P, Kostopoulos I, Bobos M, Vourli G, Samantas E, Christodoulou C, Pentheroudakis G, Pectasides D, et al. A randomized phase III study of adjuvant platinum/docetaxel chemotherapy with or without radiation therapy in patients with gastric cancer. Cancer Chemother Pharmacol. 2010;65(6):1009-21.

35. Kim KH, Kwon HC, Oh SY, Kim SH, Lee S, Kwon KA, Jang JS, Kim MC, Kim SJ, Kim HJ. Clinicopathologic significance of ERCC1, thymidylate synthase and glutathione S-transferase P1 expression for advanced gastric cancer patients receiving adjuvant 5-FU and cisplatin chemotherapy. Biomarkers. 2011;16(1):74-82.

36. Sonnenblick A, Rottenberg Y, Kadouri L, Wygoda M, Rivkind A, Vainer GW, Peretz T, Hubert A. Long-term outcome of continuous 5-fluorouracil/ cisplatin-based chemotherapy followed by chemoradiation in patients with resected gastric cancer. Med Oncol. 2012;29(5):3035-8.

37. Williams JA. Using PDX for preclinical Cancer drug discovery: the evolving field. J Clin Med. 2018;7(3):41.

38. Murphy B, Yin H, Maris JM, Kolb EA, Gorlick R, Reynolds CP, Kang MH, Keir ST, Kurmasheva RT, Dvorchik I, et al. Evaluation of alternative in vivo drug screening methodology: a single mouse analysis. Cancer Res. 2016;76(19): 5798-809.

39. Mosely SI, Prime JE, Sainson RC, Koopmann JO, Wang DY, Greenawalt DM Ahdesmaki MJ, Leyland R, Mullins S, Pacelli L, et al. Rational selection of syngeneic preclinical tumor models for immunotherapeutic drug discovery. Cancer Immunol Res. 2017;5(1):29-41.

40. Benzekry S, Lamont C, Beheshti A, Tracz A, Ebos JM, Hlatky L, Hahnfeldt P. Classical mathematical models for description and prediction of experimental tumor growth. PLoS Comput Biol. 2014;10(8):e1003800.

\section{Publisher's Note}

Springer Nature remains neutral with regard to jurisdictional claims in published maps and institutional affiliations.

Ready to submit your research? Choose BMC and benefit from:

- fast, convenient online submission

- thorough peer review by experienced researchers in your field

- rapid publication on acceptance

- support for research data, including large and complex data types

- gold Open Access which fosters wider collaboration and increased citations

- maximum visibility for your research: over $100 \mathrm{M}$ website views per year

At BMC, research is always in progress.

Learn more biomedcentral.com/submissions 